\title{
Lindblad theory of dynamical decoherence of quantum-dot excitons
}

\author{
P. R. Eastham, ${ }^{1}$ A. O. Spracklen, ${ }^{2}$ and J. Keeling ${ }^{2}$ \\ ${ }^{1}$ School of Physics, Trinity College, Dublin 2, Ireland \\ ${ }^{2}$ SUPA, School of Physics and Astronomy, University of St. Andrews, KY16 9SS, United Kingdom \\ (Received 24 August 2012; revised manuscript received 25 February 2013; published 15 May 2013)
}

\begin{abstract}
We use the Bloch-Redfield-Wangsness theory to calculate the effects of acoustic phonons in coherent control experiments where quantum-dot excitons are driven by shaped laser pulses. This theory yields a generalized Lindblad equation for the density operator of the dot, with time-dependent damping and decoherence due to phonon transitions between the instantaneous dressed states. It captures similar physics to the form recently applied to Rabi oscillation experiments [Ramsay et al., Phys. Rev. Lett. 104, 017402 (2010)] but guarantees positivity of the density operator. At sufficiently low temperatures, it gives results equivalent to those of fully non-Markovian approaches [Lüker et al., Phys. Rev. B 85, 121302 (2012)] but is significantly simpler to simulate. Several applications of this theory are discussed. We apply it to adiabatic rapid passage experiments and show how the pulses can be shaped to maximize the probability of creating a single exciton using a frequency-swept laser pulse. We also use this theory to propose and analyze methods to determine the phonon density of states experimentally, i.e., phonon spectroscopy, by exploring the dependence of the effective damping rates on the driving field.
\end{abstract}

DOI: 10.1103/PhysRevB.87.195306

PACS number(s): 78.67.Hc, 03.65.Yz, 42.50.Hz, 71.38.-k

\section{INTRODUCTION}

Controlled manipulation of coherent quantum systems is a crucial requirement for quantum information technologies, can be exploited in ultrafast switches, and may allow the exploration of exotic regimes of quantum dynamics. An important example among solid-state systems is that of excitons in quantum dots, ${ }^{1}$ which provide discrete atomic-like transitions that can be manipulated using optical pulses. These transitions have been demonstrated experimentally to correspond to twolevel systems for which resonant optical excitation induces Rabi oscillations. ${ }^{2,3}$ Thus, under pulsed excitation, the number of excitons created oscillates with the pulse area: a pulse of the correct duration and intensity creates exactly one exciton in the quantum dot, while other pulses create superpositions of one- and no-exciton states. Frequency-swept laser pulses have also been used to create single excitons in quantum dots, implementing the protocol of adiabatic rapid passage (ARP) and allowing state manipulation in a way that is robust against fluctuations in the coupling strengths and transition energies of the dots. ${ }^{4-7}$

A theoretical description of such coherent control experiments must capture both the dynamics of the driven quantum dot and the scattering and decoherence introduced by the interaction between the dot and its environment. In particular, the coupling to acoustic phonons leads to dephasing of the Rabi oscillations ${ }^{8}$ and limits the inversion in ARP. ${ }^{4,5,9,10}$ (Optic phonons could play a role in ARP with very intense, very short pulses ${ }^{11}$ where the energy scales are comparable to the optic phonon energies, but we do not consider this regime here.) The standard theoretical approach ${ }^{12}$ involves second-order perturbation theory in the phonon coupling, leading to an equation of motion for the reduced density operator of the dot. This equation involves an integral over all previous states of the dot, capturing the memory effects due to the finite bandwidth and response time of the environment.

A frequently used approach to treating this type of nonMarkovian equation of motion is a form of the Markov approximation that reduces the equation to a time-local equation, with the effects of the phonons appearing as a constant Lindblad form describing dephasing. Such an equation would be valid under the assumption that the response time of the environment is the shortest time scale in the problem. This approximation, however, is generally incorrect for quantum dot excitons. ${ }^{13-15}$ It predicts that the environment induces transitions independently of the state of the dot, in contradiction to experiments in which the driving field changes the dephasing. Such dynamical excitation-dependent dephasing has, however, been successfully described by more sophisticated approaches. These include numerically exact path integral methods ${ }^{9,10,14,16}$ (quasiadiabatic propagator path integral, QUAPI), systematic expansions in exciton-phonon correlations $^{9,10,17}$ up to fourth order and including memory effects, and time-local approximations ${ }^{8,15,18-20}$ allowing for some of the memory effects neglected in the simplest form of Born-Markov approximation. These time-local approximations improve on the simplest Born-Markov approximation by calculating the decay rates arising from system-bath coupling making use of the actual system Hamiltonian, including driving. This same point, of using the actual system Hamiltonian rather than a noninteracting approximation, is also crucial in describing the correct equilibrium state of strongly coupled systems, as discussed in Ref. 21.

When there is strong coupling to phonons but weak driving of the dot, accurate results can be found by making a polaron transformation, ${ }^{19}$ so that coupling to phonons appears in the driving term; one may then treat the driving term in the Born approximation and derive effective excitation/de-excitation rates depending on the detuning of the drive and the phonon density of states, accounting for multiphonon excitations. Such an approach can also be extended to cavity-QED situations ${ }^{13}$ with the driving replaced by a coupling to a cavity. It has also been used to study the fluorescence of a driven quantum dot, coupled to a cavity. ${ }^{22-25}$ However, the treatment of the driving within the Born approximation limits the validity of 
this approach to weak driving. Alternatively, one may view this as a statement that at strong driving, the dot state changes too rapidly for the phonons to follow, and so the polaron picture breaks down. This has been extensively studied recently ${ }^{16,19}$ using a variational polaron transformation. ${ }^{26}$ The variational approach can reproduce the exact results of the path integral across a range of driving strengths. In the limit of strong driving and experimentally relevant dot-phonon couplings, McCutcheon et al. ${ }^{16}$ show that the time-local approaches discussed above $e^{8,15,18-20}$ become increasingly accurate, as strong driving breaks the polaron picture and relatively weak dot-phonon coupling (at low temperatures) allows both a Markovian approximation and the neglect of multiphonon effects.

In this paper, we consider situations where a dot is strongly driven and with experimentally relevant (i.e., relatively weak) dot-phonon couplings. We discuss the application of the Bloch-Redfield-Wangsness ${ }^{27,28}$ (BRW) theory, widely used to describe nuclear spin relaxation, to coherent control experiments on excitons in quantum dots. It is similar to the simplest Born-Markov approximation but allows the time scale set by the inverse level spacing of the Hamiltonian to be smaller than the response time of the environment. We use this theory to derive a generalized Lindblad form for the phonon-induced damping in which the transition operators connect the time-dependent eigenstates of the dot, with the expected perturbative transition rates [see Eq. (7)]. This differs from the simplest Lindblad form mentioned above, which is often applied to quantum dots, in which the transition operators do not necessarily connect eigenstates. Furthermore, in contrast to the forms obtained by some other time-local approximations, ${ }^{15,18}$ it guarantees the positivity of the density operator and so can be used across a wider variety of pulse shapes and temperatures. We focus on the application to determining the effects of phonons for adiabatic rapid passage in quantum dots and show that the results are similar to those recently obtained from the correlation expansion at fourth order. ${ }^{9,10}$ That method includes all memory effects of the environment and allows for some phonon correlations and is known to be accurate ${ }^{9,10,29,30}$ for the parameters relevant here (since it agrees with the exact path-integral results). Thus Eq. (7) provides a simple picture of the effects of dephasing and a lightweight computational approach for modeling dephasing in quantum dots. As a further application of this theory, we use it to demonstrate the feasibility of measuring the phonon spectra and distribution functions, by exploiting the driving-field dependence of the effective damping rates. We propose and analyze two forms of such spectroscopy, one based on a generalization of the ARP experiment and one based on the response to off-resonant continuous-wave excitation.

These Markovian approximations are appropriate for strong driving; at weak driving (a limit which is avoided in the remainder of this paper), differences arise from the exact solution of the independent boson model (IBM). For infinitesimal driving, the independent boson model can be analytically solved $^{31,32}$ by finding the linear absorption spectrum about the undriven state: $A(\omega) \propto \int d t e^{-i \omega t} \exp [\varphi(t)-\varphi(0)], \varphi(t)=$ $\int d \omega e^{i \omega t} n_{B}(\omega) J(\omega) / \omega^{2}$. For the realistic phonon spectral function $J(\omega)$ considered in this paper [see Eq. (3)], the exact absorption spectrum consists of an unbroadened zero-phonon line (ZPL) and sidebands associated with one or many phonon events. At $4 \mathrm{~K}$ (the temperature considered for pulse optimization below), the ZPL contains $86 \%$ of the spectral weight. If the Markovian approach we use is applied in the limit of weak driving (outside the range of validity as discussed above), it predicts only the ZPL and misses the small phonon sidebands. The origin of this discrepancy (at 4 $\mathrm{K}$ ) is, however, not a consequence of multiphonon effects [one may safely expand the expression for $A(\omega)$ to linear order in $J(\omega)$ ] but of the Markov approximation. The origin of this discrepancy is as discussed in Refs. 33-36; Markovian approaches sample the bath at a frequency dependent on the system Hamiltonian, while absorption spectra depend on the bath response at the probe frequency. In the limit of vanishing driving, the Markovian approximation, as discussed below, produces no linewidth, hence it matches only the dominant ZPL part of the exact solution. For any nonzero driving (i.e., beyond linear response), the Markovian approach produces a nonzero linewidth, however, there is no analytic solution of the IBM for finite driving. Thus to test our theory in this regime requires comparison to numerical approaches; such a comparison to existing ${ }^{9}$ numerical results is, indeed, given below, and the match is seen to be very good (better than the match for vanishing driving), that is, as to be expected given the central point of recent work ${ }^{8,15,16,19}$ on "excitation induced dephasing", the presence of strong driving strongly affects the effective dephasing and dissipation rates, and the behavior at vanishing driving does not control how the system responds at strong driving.

The remainder of this paper is structured as follows. In Sec. II, we outline the derivation of the equation of motion for a driven quantum dot interacting with acoustic phonons. In Sec. III, we present the predictions of this equation for the inversion (exciton occupation) obtained in ARP, discuss how this process may be optimized in the presence of phonon-induced dephasing, and explain why the dephasing can in some circumstances improve the final inversion. In Sec. IV, we outline the application to phonon spectroscopy. In Sec. V, we discuss further the relationship between the positivity-preserving Lindblad form [see Eq. (7)], obtained here, and the generally positivity-violating precursor to this form [see Eq. (4)], which is sometimes used directly. ${ }^{15,18} \mathrm{We}$ present numerical results showing the failure of this latter approximation in the case of ARP pulses. In Sec. VI, we summarize our conclusions. Finally, the Appendix provides details of the derivation of Eq. (7).

\section{EQUATIONS OF MOTION}

In this section, we present the derivation of the secularized (Lindblad) form of the equation of motion for the reduced density matrix $\rho(t)$. Our approach initially follows the same steps as in Refs. 15 and 18. However, in order to produce an approach that preserves positivity of the density matrix throughout the range of validity of perturbation theory, we must additionally secularize the resulting equations. ${ }^{37,38}$ For completeness, we include in this paper also the steps that follow Refs. 15 and 18. In this section, we outline the main steps of the derivation, and present further details in the Appendix. 
We consider a single quantum dot, driven close to one of its discrete transition frequencies by a laser pulse with a time-dependent amplitude and frequency. For simplicity, we assume that the excitation is circularly polarized, so that only one of the exciton spin states is relevant and we may neglect the formation of biexcitons. Thus we model the dot as a two-level system, which may be in the ground $|0\rangle$ or the one-exciton $|X\rangle$ state. The Hamiltonian may be expressed, using pseudospin$1 / 2$ operators $\mathbf{s}$, as $(\hbar=1)$

$$
H_{\mathrm{dot}}=\Delta(t) s_{z}-\Omega(t) s_{x},
$$

in the rotating-wave approximation and in a frame rotating at the instantaneous driving frequency $\omega(t)$. Here, $\Delta(t)=$ $E_{0}-\omega(t)$ is the detuning between the transition energy $E_{0}$ and the instantaneous driving frequency. $\Omega(t)$ is the timedependent Rabi frequency, corresponding to the amplitude of the driving pulse. $\mathbf{s}=\sigma / 2$, where the Pauli operator $\sigma_{z}=$ $|X\rangle\langle X|-| 0\rangle\langle 0|$ describes the occupation of the exciton state, while $\sigma_{x}=|X\rangle\langle 0|+| 0\rangle\langle X|=s_{+}+s_{-}$describes the electric dipole moment of the transition. We refer to $s_{z}=\sigma_{z} / 2$ as the inversion.

We focus on the effects of acoustic phonons, which are the dominant dephasing mechanism in recent Rabi flopping ${ }^{15,18}$ and ARP experiments. ${ }^{4,5,9,10}$ They couple to the dot via the deformation potential coupling,

$$
H_{\mathrm{c}}=s_{z} \sum_{q}\left(g_{q} b_{q}+g_{q}^{*} b_{q}^{\dagger}\right),
$$

where $q$ labels the phonon wave vectors, $b_{q}\left(b_{q}^{\dagger}\right)$ is a phonon annihilation (creation) operator, and $g_{q}$ is the coupling constant. The phonon effects are controlled by the phonon spectral density, $J(\omega)=\sum_{q}\left|g_{q}\right|^{2} \delta\left(\omega-\omega_{q}\right)$. We take the model used in Ref. 15 for a GaAs/InGaAs quantum dot,

$$
J(\omega)=\frac{\hbar A}{\pi k_{B}} \omega^{3} e^{-\omega^{2} / \omega_{c}^{2}},
$$

with similar parameters $A=11.2$ fs $\mathrm{K}^{-1}, \hbar \omega_{c}=2 \mathrm{meV}$. In Eq. (3), the low-frequency behavior $J(\omega) \propto \omega^{3}$ arises from the coupling and density of states for acoustic phonons, while the high-frequency cutoff at $\omega_{c}$ arises from the size of the dot; confined excitons do not couple effectively to phonons of wavelengths smaller than the confinement.

In the limit that $\Delta(t)$ and $\Omega(t)$ vary slowly with time, we may treat them effectively as constants and use the approach discussed in Refs. 15 and 18, so that the effect of the acoustic phonons can be found by transforming to the interaction picture and using the Born-Markov approximation there. This requires that the interaction picture density operator $\tilde{\rho}(t)$ is approximately constant over the correlation time of the phonon bath $\left(\sim 1 / \omega_{c}\right)$, so that $\tilde{\rho}\left(t^{\prime}\right) \simeq \tilde{\rho}(t)$ on the right-hand side of Eq. (A4). Equivalently, this means that the bath density of states should be flat over the effective linewidth of the system, as illustrated in Fig. 1. Such an approximation is valid as long as neither the decay rate nor sweep rates $(\dot{\Delta} / \Delta, \dot{\Omega} / \Omega)$ are too high, as both contribute to the effective linewidth. Note that applying the Born-Markov approximation directly in the Schrödinger picture requires additionally that the density of states is flat on the scale set by the position of the line, i.e., the energy scale of the Hamiltonian, and this is not the case here.

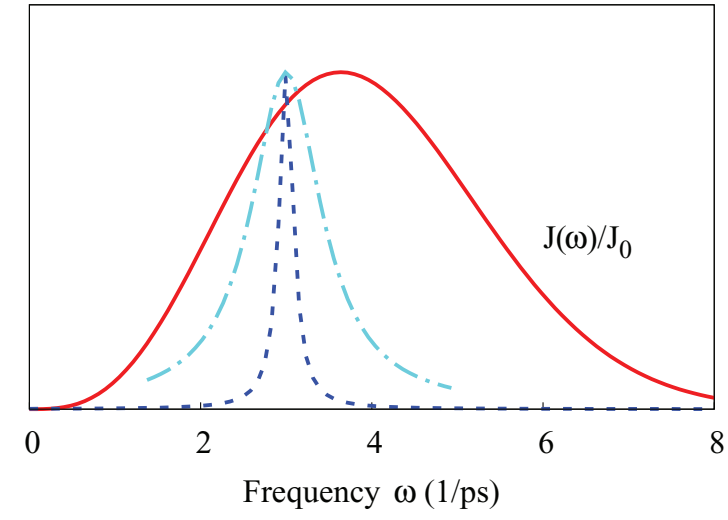

FIG. 1. (Color online) Illustration of time scales required for validity of (time-dependent) Markov approximation, shown in the frequency domain. Solid red line shows the frequency-dependent decay rate, illustrated by the phonon spectral function $J(\omega)$. At low temperatures and slow driving (dashed line), the linewidth is sufficiently small that the decay rate does not vary significantly across the linewidth (bath correlation time is short compared to decay time). At higher temperatures or faster driving, the linewidth grows (dot-dashed line) so that the decay rate does vary (decay time or sweep time become comparable to bath correlation time). The lineshapes illustrated are the Lorentzians corresponding to transition rates $T_{1}^{-1}=0.2$ and $1.0 \mathrm{ps}^{-1}$, which may be compared with Fig. 2 .

It is convenient to introduce rotated spin operators $\mathbf{r}=R \mathbf{s}$, with $R$ a rotation by angle $\tan ^{-1} \Omega / \Delta$ around the $y$ axis, so that the instantaneous system Hamiltonian becomes $H_{\mathrm{dot}}=\Lambda r_{z}$ where $\Lambda=\sqrt{\Omega^{2}+\Delta^{2}}$ is the dressed-state splitting. For the acoustic phonon coupling considered here, this yields (see Appendix for further details)

$$
\dot{\tilde{\rho}}=-P Q \tilde{\rho}+Q \tilde{\rho} P+P \tilde{\rho} Q^{\dagger}-\tilde{\rho} Q^{\dagger} P,
$$

where $P$ and $Q$ are time-dependent operators of the form

$$
\begin{aligned}
P(t)= & \frac{\Delta}{\Lambda} r_{z}+\frac{\Omega}{2 \Lambda}\left(r_{+} e^{i \Lambda t}+r_{-} e^{-i \Lambda t}\right), \\
Q(t)= & \int d \nu J(\nu) \int^{t} d t^{\prime} P\left(t^{\prime}\right) \\
& \times\left[\left(n_{\nu}+1\right) e^{-i \nu\left(t-t^{\prime}\right)}+n_{\nu} e^{i \nu\left(t-t^{\prime}\right)}\right],
\end{aligned}
$$

and $n_{v}$ is the phonon occupation function at frequency $v$. After undoing the transformation to the interaction picture, this gives the density matrix equation form corresponding to the results in Ref. 18.

This equation is not of Lindblad form, and consequently, it can lead to density matrix evolution that violates positivity. For the relatively short pulses in Refs. 15 and 18, one may readily check that this is not a problem. However, for our application to ARP pulses, positivity violation can occur at late times under conditions where the perturbative approximations required for Born-Markov remain valid; this is discussed further in Sec. V.

This issue of positivity violation was discussed extensively in, e.g., Ref. 37, where it was shown that there exists more than one form of Markovian density matrix equation that faithfully represent the infinitesimal increment of the full density matrix evolution in the Markovian (perturbative) 
limit. However, although these different forms are equivalent regarding infinitesimal time steps, those equations that are not of Lindblad form do not conserve positivity. A Lindblad form can nonetheless be derived by averaging so as to remove the rapidly oscillating terms in Eq. (4)—such a procedure, known as secularization, yields a Lindblad form that in the perturbative limit is equally valid to Eq. (4). Further details are presented in the Appendix. After transforming back to the Schrödinger picture, the result is

$$
\begin{aligned}
\dot{\rho}= & -\left[\gamma_{a}(\Omega, \Delta) / 2\right]\left(r_{-} r_{+} \rho+\rho r_{-} r_{+}-2 r_{+} \rho r_{-}\right) \\
& -\left[\gamma_{e}(\Omega, \Delta) / 2\right]\left(r_{+} r_{-} \rho+\rho r_{+} r_{-}-2 r_{-} \rho r_{+}\right) \\
& -i\left[r_{z}, \rho\right] \Delta E(\Omega, \Delta)-i\left[H_{\mathrm{dot}}, \rho\right],
\end{aligned}
$$

where we have made explicit the time dependence of the decay rates due to the dependence on the slow variation of the parameters $\Omega(t)$ and $\Delta(t)$. This is a time-dependent generalization of the standard form ${ }^{27}$ obtained from the secularized Born-Markov approximation in the interaction picture, as used in some related contexts. ${ }^{39,40}$ In addition to now preserving positivity, it makes explicit the origin and nature of decay terms; the damping appears as a Lindblad form describing transitions between the instantaneous dressed states, with the phonon absorption and emission rates

$$
\begin{gathered}
\gamma_{a}=2\left(\frac{\Omega}{2 \Lambda}\right)^{2} \pi J(\Lambda) n(\Lambda), \\
\gamma_{e}=2\left(\frac{\Omega}{2 \Lambda}\right)^{2} \pi J(\Lambda)[n(\Lambda)+1] .
\end{gathered}
$$

These rates are shown in Fig. 2 for the spectral function (3).

In addition, Eq. (7) includes a phonon Lamb shift (see Fig. 3): the energy splitting of the dressed states is now

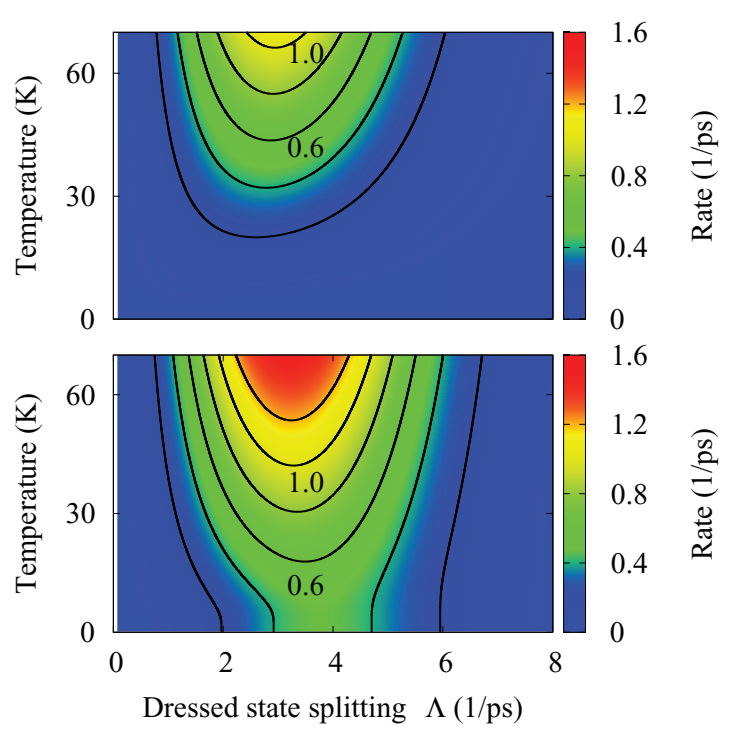

FIG. 2. (Color online) Phonon absorption (top) and emission (bottom) rates, Eqs. (8) and (9), respectively, divided by the squared ratio of Rabi splitting to dressed-state energy splitting, $\Omega^{2} / \Lambda^{2}$, as a function of the dressed-state energy splitting $\Lambda=\sqrt{\Omega^{2}+\Delta^{2}}$; for resonant driving $\Delta=0$ and the scaling is one.

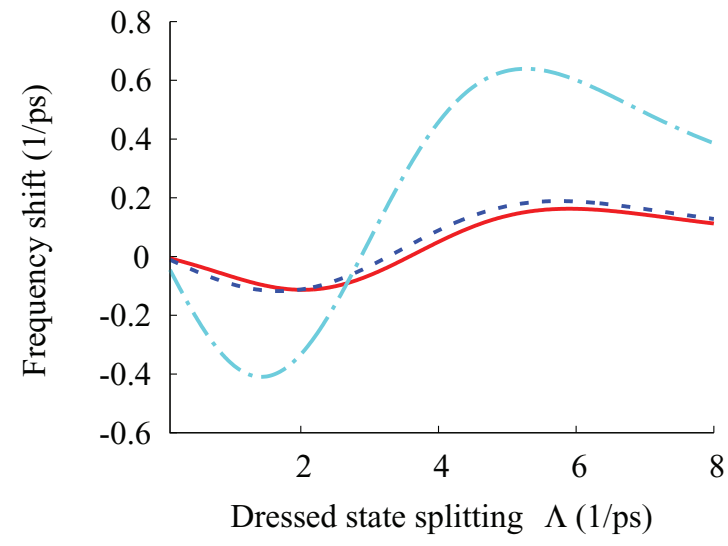

FIG. 3. (Color online) Phonon-induced change in the dressedstate splitting $\Delta E$ [see Eq. (10)] divided by the squared ratio of Rabi splitting to dressed-state energy splitting, i.e., $\Delta E /\left(\Omega^{2} / \Lambda^{2}\right)$, as a function of the dressed-state energy splitting $\Lambda$. Curves are temperatures 1 (solid), 10 (dashed), and $50 \mathrm{~K}$ (dot-dashed).

$\Lambda+\Delta E$ with

$$
\Delta E=-\left(\frac{\Omega}{2 \Lambda}\right)^{2} 2 \Lambda \int \frac{J(\nu) \operatorname{coth}(\nu / 2 k T)}{\nu^{2}-\Lambda^{2}} d \nu .
$$

Figure 2 may be used to establish the validity of Eq. (7), by comparing the decay rates with the extent of their frequency dependence. We see that at the highest temperatures, the shown peak damping rates, and therefore the linewidths, can become a significant fraction of the width of the spectral function, as illustrated in Fig. 1. In this regime, the Markovian approximation that $\tilde{\rho}(t)$ varies slowly on the time scale $1 / \omega_{c}$ breaks down, and a quantitative analysis requires the solution of a nonlocal equation. ${ }^{9,10}$ However, as can be seen from the linewidths in Fig. 1, we expect qualitatively reasonable results over much of the parameter regimes shown, and the approximations should be quantitatively accurate in the low-temperature regime, below $20 \mathrm{~K}$, typical of most coherent control experiments. We note that the Markov approximation amounts to approximating the spectral function with its constant value at the dressed frequency $\Lambda$. At high temperatures, the damping at any one splitting will sample a finite range of the spectral function, so that we expect a weaker dependence of the effective linewidth on the energy splittings than indicated here, as well as non-Lorentzian emission lines. Similarly, we expect the Markov approximation to overestimate the Lamb shift and its frequency dependence at high temperatures.

As well as becoming invalid at high temperatures, where the effective linewidth becomes large due to scattering, the approximations used above also fail if the time dependence of the parameters $\Delta(t)$ and $\Omega(t)$ becomes too strong. This is due to the finite bandwidth $1 / \tau_{\text {chirp }} \sim \dot{\Delta} / \Delta$ arising from the time dependence of the parameters. Alternatively, one may understand this as arising from the fact that for small enough $\tau_{\text {chirp }}$, the bath correlation time no longer is the shortest time scale in the problem.

The density matrix evolution described above is a complete description of the time evolution of the system. In some cases, it can be useful to write this in an alternative representation, by considering the time evolution of the components of the Bloch 
vector, which we write here for completeness:

$$
\begin{aligned}
\dot{s}_{x}= & -\frac{\Omega}{2 \Lambda}\left(\gamma_{a}-\gamma_{e}\right)-\left[\frac{\Delta^{2}+2 \Omega^{2}}{2 \Lambda^{2}}\left(\gamma_{a}+\gamma_{e}\right)\right] s_{x} \\
& -\Delta s_{y}+\frac{\Delta \Omega}{2 \Lambda^{2}}\left(\gamma_{a}+\gamma_{e}\right) s_{z}, \\
\dot{s}_{y}= & \Delta s_{x}-\left(\gamma_{a}+\gamma_{e}\right) s_{y} / 2+\Omega s_{z}, \\
\dot{s}_{z}= & \frac{\Delta}{2 \Lambda}\left(\gamma_{a}-\gamma_{e}\right)+\frac{\Delta \Omega}{2 \Lambda^{2}}\left(\gamma_{a}+\gamma_{e}\right) s_{x}-\Omega s_{y} \\
& -\left[\frac{2 \Delta^{2}+\Omega^{2}}{2 \Lambda^{2}}\left(\gamma_{a}+\gamma_{e}\right)\right] s_{z} .
\end{aligned}
$$

Note that here we have neglected the small Lamb shift.

\section{INVERSION IN ARP}

The above results apply in general to any time-dependent pulse sequence. We can in particular consider pulses corresponding to adiabatic rapid passage in which the detuning $\Delta(t)$ is swept smoothly through zero with the intention of creating a one-exciton state. Under such a pulse, there is an avoided crossing between the zero- and one-exciton states of Eq. (1), generated by the driving field. We aim to adiabatically follow a single energy level, thus evolving from the initial ground state to the one exciton state. Acoustic phonon effects in this process have already been explicitly considered in Refs. 9 and 10, for fixed-bandwidth pulses of the form

$$
\begin{aligned}
& \Delta(t)=-\frac{a t}{\left(a^{2}+\tau_{0}^{4}\right)}, \\
& \Omega(t)=\frac{\Theta_{0}}{\sqrt{2 \pi \sqrt{a^{2}+\tau_{0}^{4}}}} \exp \left[-\frac{t^{2} \tau_{0}^{2}}{2\left(a^{2}+\tau_{0}^{4}\right)}\right],
\end{aligned}
$$

where $\Theta_{0}$ is the area of the bandwidth-limited pulse before the chirp is applied, and $a$ is the spectral chirp. ${ }^{41}$ The results obtained from Eq. (7) for this form of pulse, plotted on a similar scale to those in Fig. 2 of Ref. 9, are shown in Fig. 4. As expected from the discussion above, the results are very similar at low temperatures and slow sweep rates, where the conditions for the Born-Markov approximation are well satisfied.

As discussed in Ref. 9, the asymmetry about the line $a=0$ at low temperatures arises because absorption processes can be neglected $\gamma_{a} \ll \gamma_{e}$ for $T \ll \omega_{c}$, and as is clear from Eq. (7), emission can only occur when the dot is in the higher-energy dressed state. For $a<0$, the ARP protocol attempts to follow this higher-energy dressed state, so that the process is susceptible to phonon emission, whereas for $a>0$ it is not. The simplicity of Eq. (7) allows one to further see that for the range plotted, the values of $\Lambda(t=0)=\Omega(t=0)$ lie below the peak of scattering rates (see Fig. 2), hence the decrease of inversion with increasing pulse area visible within Fig. 4. For larger pulse areas, the central value of $\Lambda$ can exceed this peak (at $\Theta_{0} \approx 6 \pi$ for $a=0$ ) and inversion then increases with pulse area.

\section{A. Optimization of ARP}

The relatively lightweight effort of simulating Eq. (7) allows one to rapidly investigate the effects of other potential pulse shapes and ARP protocols beyond that in Eq. (12). In
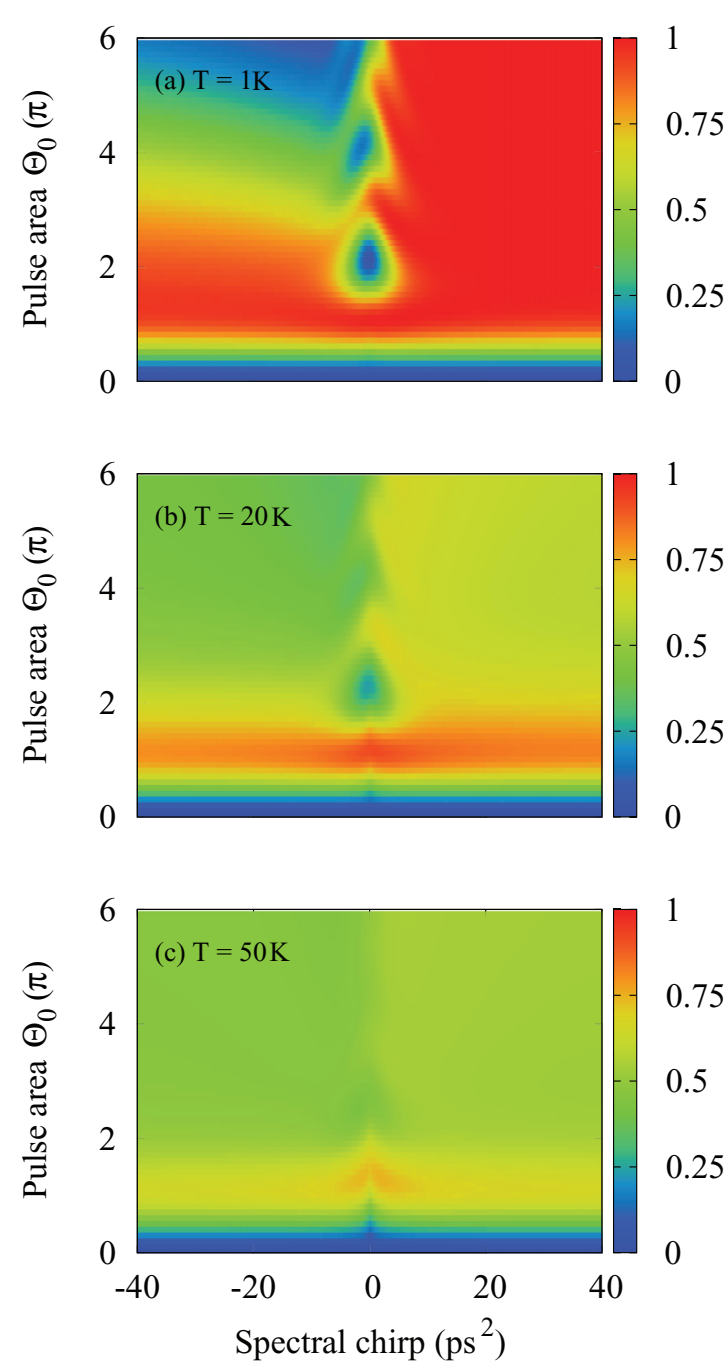

FIG. 4. (Color online) Final exciton occupation probability following an ARP pulse of the form given in Eq. (12), calculated using the time-dependent Lindblad form given in Eq. (7). Calculated using $\tau_{0}=2 \mathrm{ps}$, at temperatures 1 (a), 20 (b) and $50 \mathrm{~K}$ (c), for direct comparison with Fig. 2 of Ref. 9. For low temperatures and slow sweeps, the results are very similar; for larger temperatures and higher chirp rates, the limited Markov approximation used in deriving Eq. (7) becomes invalid.

the absence of decay, the question of how the final excited state population can be optimized for a given pulse area has been extensively studied by Guérin et al. ${ }^{42}$ By considering the leading order nonadiabatic effects, they showed that these were minimized in the case where $\Lambda(t)$ was independent of time. This implies that in the limit of large pulse areas (deep in the adiabatic regime), maximum excitation should be reached when the chirp rate is adjusted to match this condition.

The time dependence of $\Delta$ and $\Omega$ given in Eq. (12) cannot achieve a time-independent $\Lambda$. Instead, other pulse shapes need to be considered, such as

$$
\Delta(t)=-\Delta_{0} \tanh \left(\frac{t}{\tau}\right), \quad \Omega(t)=\Omega_{0} \operatorname{sech}\left(\frac{t}{\tau}\right),
$$

which have a pulse area $\Theta$ when $\Omega_{0}=\Theta / \tau$. As discussed by Guérin et al. ${ }^{42}$ the optimum condition $\Omega_{0}=\Delta_{0}$ arises 


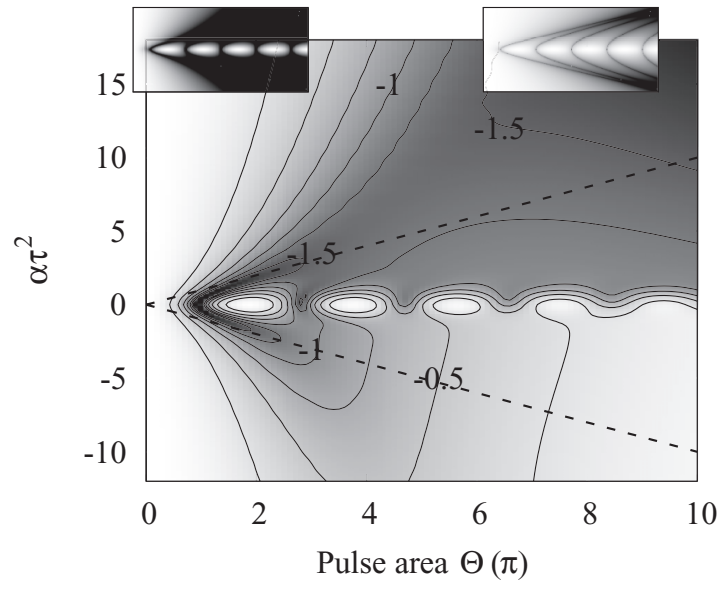

FIG. 5. Final exciton occupation probability for the sech pulse [see Eq. (13)] with $\Omega_{0}=\Theta / \tau$ and $\Delta_{0}=\alpha \tau$ for $\tau=6$ ps at a temperature $4 \mathrm{~K}$. The gray scale and contour labels show $\log _{10}(1-$ $P$ ), where $P$ is the final occupation probability. Insets show the case without phonon induced dephasing, as in Ref. 42, on the same gray scale (from -2 to 0 , left inset) and on a gray scale with a larger range (from -10 to 0 , right inset). Main panel shows the effects of dynamical dephasing on the same pulse shape. Dashed line indicates the condition $\Omega_{0}=\Delta_{0}$, which gives the optimal transfer in the absence of dephasing.

from the convergence of lines originating from the maxima of the Rabi oscillations. This is shown in the inset of Fig. 5. However, although such pulses are optimal in the isolated case, the differences in excitation near this line are exponentially small and entirely dwarfed by the effects of phonon induced dephasing. In the presence of dephasing, not only are the sharp, exponentially small features washed out, but the optimum chirp rate $\Delta_{0} / \tau$ moves to significantly larger values, due to the reduction of the dephasing rate at large $\Lambda$.

\section{B. Thermalization enhanced inversion}

The discussion of the effects of acoustic phonons so far has been in terms of their reducing the final state inversion as compared to near-perfect inversion achieved deep in the adiabatic regime. There exists, however, a significant range of experimental conditions for which coupling to phonons can instead enhance the final state inversion. This effect has recently been discussed by Reiter et al. ${ }^{10}$ in the context of compensating for detuning of quantum dots. Even in the absence of detuning, coupling to phonons can enhance the final state inversion. An increase in the efficiency of adiabatic transfer due to damping processes has also recently been reported for a many-boson model. ${ }^{43}$

Figure 6 illustrates this potential enhancement by showing how the final inversion is affected if the decay rates are rescaled by a factor $q$, i.e., $J(\omega) \rightarrow q J(\omega)$, considering a pulse shape:

$$
\Omega=\Omega_{0} \operatorname{sech}\left(\frac{t}{\tau}\right), \quad \Delta=-\alpha t .
$$

For a wide range of parameters $\Theta$ and $\alpha$ the dependence on $q$ is nonmonotonic: small coupling to phonons decreases the inversion, but further increase in coupling then increases final inversion. This nonmonotonic behavior [shown in Fig. 6(a)]
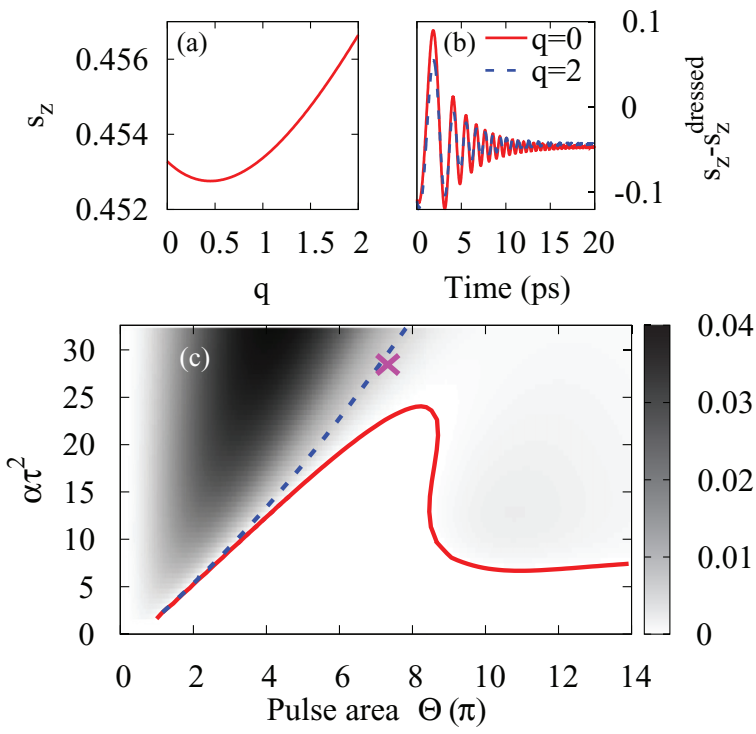

FIG. 6. (Color online) Dependence of inversion on strength of phonon coupling in ARP. (a) indicates how for a specific value of $\alpha$ and $\Omega_{0}$, there is a nonmonotonic dependence of inversion upon phonon coupling. (b) Time evolution of $s_{z}$ at two values of $q$, relative to its ideal value for adiabatically following the dressed states. (c) Maps where nonmonotonic dependence on $q$ arises. This is determined by two quantities: the gray scale indicates the value of $\operatorname{Max}_{0<q<2}\left(d s_{z} / d q\right)$, and the solid red line is the boundary where this is zero. The blue dashed line indicates where $d s_{z} /\left.d q\right|_{q=0}=0$. Between these lines, nonmonotonic dependence as seen in (a) occurs. The magenta cross indicates the conditions used for (a) and (b). $T=4 \mathrm{~K}$, $\tau=5.68$ ps.

exists throughout the region between the solid and dashed lines in Fig. 6(c). For large chirp rates and weak pulses, the inversion without coupling to phonons is already poor, and coupling to phonons increases the inversion; this corresponds to the behavior above the dashed line in Fig. 6(c).

The origin of this enhancement at large $q$ can be understood by considering that for large $q$, the quantum dot state will come to thermal equilibrium with the phonon bath. Since the coupling to phonons depends on the prefactor $q(\Omega / \Lambda)^{2}$, the coupling to phonons will eventually switch off as $\Omega \rightarrow 0$. However, the larger the value of $q$, the later this switch off occurs, and so the longer the system maintains thermal equilibrium with phonons. As the detuning $\Delta$ continues to increase at late times, the inversion of this equilibrated state therefore increases with increasing $q$.

\section{PHONON SPECTROSCOPY}

A second application of the relative simplicity of Eq. (7) is to see how the phonon density of states can be recovered from spectroscopy using an appropriately designed pulse sequence. This would, in principle, allow direct experimental confirmation of the model phonon coupling $J(\omega) \propto \omega^{3} \exp \left(-\omega^{2} / \omega_{c}^{2}\right)$ as widely used ${ }^{8,15,18}$ in modeling quantum dots. In the following, we present and compare two approaches to this, based on either short-time or long-time behavior, incorporating spontaneous decay in the long-time process. 


\section{A. Modified ARP protocol}

The short-time approach uses a modified version of an ARPlike pulse sequence. We consider an ARP protocol divided into pieces, so that the sweep of $\Delta(t)$ is interrupted by a wait period $T_{w}$ at a value $\Delta_{w}$ before completing the ARP sweep. The initial and final sweeps serve to map between initial or final eigenstates of $s_{z}$ and eigenstates of $r_{z}$ during the wait period. The final inversion depends on the effect of the phonons during the wait time, sampling the phonon density of states at a frequency $\Lambda_{w}=\sqrt{\Delta_{w}^{2}+\Omega_{w}^{2}}$. During this wait time, Eq. (7) reduces to $\dot{p}_{\downarrow}=-\gamma_{a} p_{\downarrow}+\gamma_{e} p_{\uparrow}, \dot{p}_{\uparrow}=\gamma_{a} p_{\downarrow}-\gamma_{e} p_{\uparrow}$ in terms of the diagonal elements in the $\mathbf{r}$ basis. The deviation from inversion of the final state after a given wait time $T_{w}$ is thus given by

$$
\frac{1}{2}-s_{z} \simeq \frac{\gamma_{a, e}}{\gamma_{a}+\gamma_{e}}\left[1-e^{-\left(\gamma_{a}+\gamma_{e}\right) T_{w}}\right]
$$

where $\gamma_{a, e}=\gamma_{a, e}\left(\Omega_{w}, \Delta_{w}\right)$, the numerator of the right-hand side is $\gamma_{a}$ for a forward sweep ( $\Delta$ decreasing with time), and $\gamma_{e}$ for a reverse sweep ( $\Delta$ increasing with time). For long wait times, the excitations reach thermal equilibrium with the phonon bath, as expected ${ }^{21}$ and so $s_{z}$ becomes independent of the phonon density of states. For short wait times $\left(\gamma_{a}+\gamma_{e}\right) T_{w} \ll 1$, one finds $\frac{1}{2}-s_{z} \simeq \gamma_{a, e}\left(\Omega_{w}, \Delta_{w}\right) T_{w}$, thus by varying $\Omega_{w}$ one can directly map out the damping rate.

In order to extract the phonon density of states with some accuracy, the pulse sequence must be carefully chosen. At low temperatures, $\gamma_{a} \ll \gamma_{e}$, and so spectroscopy using the forward sweep is hard to achieve- the reduction in inversion is tiny for times $T_{w}$ such that $T_{w}\left(\gamma_{a}+\gamma_{e}\right) \lesssim 1$ and tends to be dwarfed by effects of nonadiabaticity. Using a "reverse" ARP pulse produces a clearer signal. However, in order to have the signal dominated by the waiting time, it is necessary for the wait time to be longer than the sweep, and the sweep to be sufficiently slow for all values of $\Omega_{w}$. For this to be compatible with $T_{w} \gamma_{e} \lesssim 1$, it is helpful to choose $\Delta_{w} \neq 0$ so that the rate $\gamma_{e}$ is suppressed by a factor $\left(\Omega_{w} / \Lambda_{w}\right)^{2}<1$. Combining these considerations, the pulse sequence

$$
\begin{aligned}
& \Delta(t)=\Delta_{w}+\Delta_{0}\left[\tanh \left(\frac{t+t_{\Delta}}{\tau_{\Delta}}\right)+\tanh \left(\frac{t-t_{\Delta}}{\tau_{\Delta}}\right)\right], \\
& \Omega(t)=-\frac{\Omega_{w}}{2}\left[\tanh \left(\frac{t+t_{\Omega}}{\tau_{\Omega}}\right)-\tanh \left(\frac{t-t_{\Omega}}{\tau_{\Omega}}\right)\right]
\end{aligned}
$$

gives the results shown in Fig. 7 with parameter values given in the figure caption.

There is a reasonable match between the prediction of Eq. (15) for decay during the wait time and the actual inversion, but the match is not perfect due to the effects of phonons during the initial and final sweep, as well as some remaining degree of nonadiabaticity (at small $\Omega_{w}$ ). Nonetheless, one may invert Eq. (15) in order to extract the phonon density of states from the measured inversion, and the result of this procedure is shown as the dot-dashed line in the inset of Fig. 8. The reasonable agreement confirms that for such a pulse the final inversion contains sufficient information to extract the phonon density-of-states; in practice, one might include corrections to Eq. (15) by comparing an experiment directly to Eq. (7).

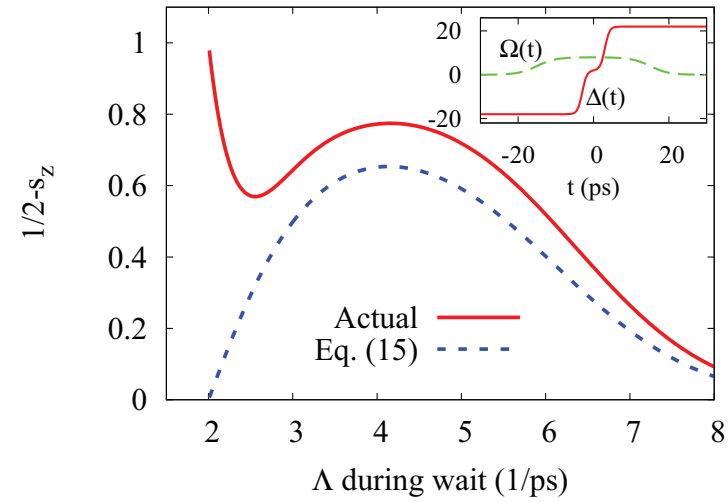

FIG. 7. (Color online) Phonon spectroscopy using divided ARP pulse. Main figure: deviation from inversion following the protocol in Eq. (16) (solid), compared with the approximation of Eq. (15) (dashed line), plotted by varying $\Omega_{w}$. Inset: time dependence of $\Delta(t)$ (solid) and $\Omega(t)$ (dashed), with parameters $\Delta_{w}=2 \mathrm{ps}^{-1}, \Delta_{0}=-10 \mathrm{ps}^{-1}, t_{\Delta}=T_{w} / 2=3 \mathrm{ps}, \tau_{\Delta}=$ $1.2 \mathrm{ps}, t_{\Omega}=15 \mathrm{ps}, \tau_{\Omega}=5 \mathrm{ps}$, and $T=4 \mathrm{~K}$.

\section{B. Steady state of driven open system}

An alternate approach to reconstructing the phonon density of states arises by considering the long-time behavior, allowing for both spontaneous decay as well as coupling to acoustic phonons, i.e.,

$$
\dot{\rho} \rightarrow \dot{\rho}+(\kappa / 2)\left(s_{+} s_{-} \rho+\rho s_{+} s_{-}-2 s_{-} \rho s_{+}\right) .
$$

Equivalently, such loss modifies the equations for the Bloch vector components as follows:

$$
\frac{d}{d t}\left(\begin{array}{c}
s_{x} \\
s_{y} \\
s_{z}
\end{array}\right) \rightarrow \frac{d}{d t}\left(\begin{array}{c}
s_{x} \\
s_{y} \\
s_{z}
\end{array}\right)+\kappa\left(\begin{array}{c}
s_{x} / 2 \\
s_{y} / 2 \\
s_{z}+1 / 2
\end{array}\right)
$$

In particular, we consider the long-time behavior under a constant driving, $\Omega(t)=\Omega_{0}, \Delta(t)=\Delta_{0}$. In this case, the longtime behavior should be understood as a steady state arising from the balance of coupling to the phonon reservoir and the

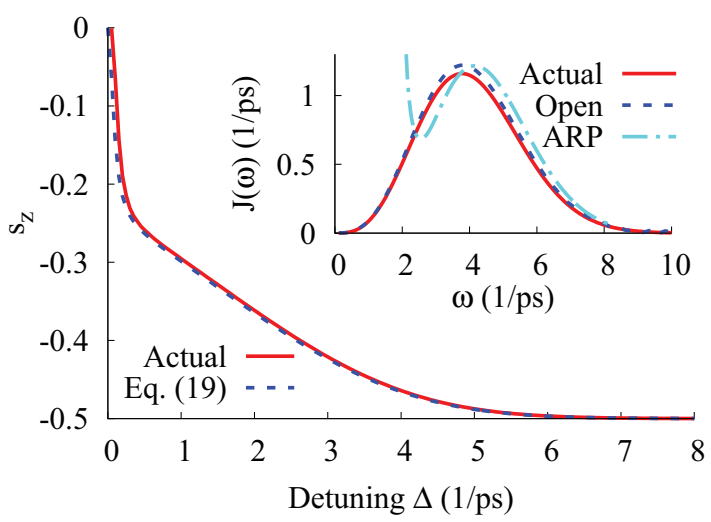

FIG. 8. (Color online) Steady-state value of $s_{z}$ with driving at $\Omega=$ $0.1 \mathrm{ps}^{-1}$, with a spontaneous decay rate of $\kappa=2 \mathrm{~ns}^{-1}$, as a function of the detuning, at $T=20 \mathrm{~K}$. (Inset) Comparison of actual phonon density of states $J(\omega)$ with the values reconstructed by inverting Eq. (19) and from the modified ARP approach, inverting Eq. (15) (parameters for modified ARP as in Fig. 7). 
decay due to coupling to a photon reservoir. The pumping term $\Omega$ is necessary only in order to enable the phonon coupling to create and destroy excitations of the quantum dot. In the absence of spontaneous decay terms (no photon reservoir), the steady state would trivially be the thermal equilibrium of the system Hamiltonian, due to equilibration with the phonon reservoir. In this case, the phonon density of states does not appear, and only the phonon temperature matters. However, the coupling to the photon reservoir drives the system into a nonthermal steady state in which the balance of spontaneous decay rate and phonon coupling determines the state. One may then read out the phonon density of states from the steady-state inversion achieved.

In the limit of small decays, i.e., $\kappa, \gamma_{a}, \gamma_{e} \ll \Omega, \Delta$, one may analytically solve the equations for the Bloch vector components, given in Eqs. (11) and (18), by expanding the steady-state equations with respect to the decay rates. In the limit of vanishing decay terms $\kappa, \gamma_{a}, \gamma_{e} \rightarrow 0$, it is clear that the steady state of Eq. (11) requires $\Delta s_{x}+\Omega s_{z}=0, s_{y}=0$. Then, including the decay terms to first order, one finds $s_{y} \sim$ $\mathcal{O}\left(\kappa, \gamma_{a}, \gamma_{e}\right)$, and so one may continue to use $s_{x}=-(\Omega / \Delta) s_{z}$ as the solution of $\dot{s}_{y}=0$ up to order $\mathcal{O}\left(\kappa^{2}, \gamma_{a}^{2}, \gamma_{e}^{2}\right)$. Using this, the remaining two equations $\dot{s}_{x, z}=0$ can be solved by eliminating $s_{y}$, to give

$$
s_{z}=\frac{-\Delta^{2} \kappa+\Lambda \Delta\left(\gamma_{a}-\gamma_{e}\right)}{\left(\Omega^{2}+2 \Delta^{2}\right) \kappa+2 \Lambda^{2}\left(\gamma_{a}+\gamma_{e}\right)} .
$$

As anticipated, this expression involves the ratio of phonon and spontaneous decay terms. In the limit $\kappa \rightarrow 0$, one recovers the thermal equilibrium result so that $s_{z}=(-\Delta / 2 \Lambda)\left(\gamma_{e}-\right.$ $\left.\gamma_{a}\right) /\left(\gamma_{a}+\gamma_{e}\right)$ in which case the phonon density of states cancels, and no information about the phonon bath (other than temperature) appears in $s_{z}$. However, for $\kappa \neq 0$, the steady state depends on the ratio of $\gamma^{a} / \kappa, \gamma^{e} / \kappa$, and this in turn allows the phonon density of states to be extracted from the final state.

Figure 8 shows how the phonon density of states can be reconstructed by extracting the steady state for a fixed value of $\Omega$, and varying the pump detuning $\Delta$. By inverting Eq. (19), and assuming the phonon temperature is known, one may extract the effective phonon density of states as shown in the inset. The density of states reconstructed this way matches the actual density of states used in the density matrix evolution very closely. It may also be possible to use recently developed variational approaches ${ }^{16}$ to extend such phonon spectroscopy to more strongly coupled systems.

\section{LINDBLAD VERSUS NON-LINDBLAD APPROXIMATIONS}

As mentioned above, the question of which approximate Markovian density matrix equation best corresponds to a given full density matrix equation was discussed extensively by Dumcke and Spohn. ${ }^{37}$ The conclusion there is that in the limit of short bath correlation times, there exist multiple approximate Markovian equations that have the same order of validity, as defined by the limiting behavior of $\left\|\rho^{\text {approx }}-\rho^{\text {full }}\right\|$ (where $\|\cdots\|$ is the trace norm) as the coupling to the bath vanishes. In other words, there are several approximations that give the same results for the short time scales over which perturbation theory applies. These different approximate Markovian equations differ in regard of whether or not one averages over rapidly oscillating terms in the interaction picture, explicitly eliminating terms that are in any case negligible in the limit where perturbation theory applies. Without such averaging, we reach Eq. (4), while averaging leads instead to Eq. (7). However, positivity of the density matrix is only preserved for the Lindblad form in Eq. (7), and so Dumcke and Spohn ${ }^{37}$ conclude that only this approach is correct.

In general, these rapidly oscillating time-dependent terms give small changes in the density operator over small time intervals. However, problems can arise when we use the Markov approximation to join together many such small time intervals, and evolve the density operator over long times. If the time-dependent terms are not treated consistently with the Markov approximation, it might lead to an unphysical growth of these small corrections and potentially unphysical results. Indeed, we note that retaining the rapidly oscillating terms is formally inconsistent with the Markov approximation of replacing $\tilde{\rho}\left(t^{\prime}\right) \rightarrow \tilde{\rho}(t)$ in Eq. (A4): this assumes that $\tilde{\rho}\left(t^{\prime}\right)$ varies more slowly than the remainder of the integrand, and in particular more slowly than the time scale $1 / \Lambda$.

It is often assumed that such a point is irrelevant as small decay rates (as are required for validity of the Markov approximation) imply that any possible violation of positivity is negligible. However, for problems involving weak decay and long time evolution, such as the current problem, positivity violation can occur for Eq. (4) even in regimes where the Markov approximation appears to be valid. This can indeed lead to unphysical results, as shown, for example, in Figs. 9 and 10. We note that for the Rabi oscillations studied by Ramsay et al. ${ }^{15}$ no positivity violation occurs for the parameters used and the results of Eqs. (4) and (7) are hardly distinguishable; indeed, these equations can give similar results for ARP pulses, as can be seen by comparing Fig. 10 [which shows the results of the non-Lindblad Eq. (4)] to Fig. 4

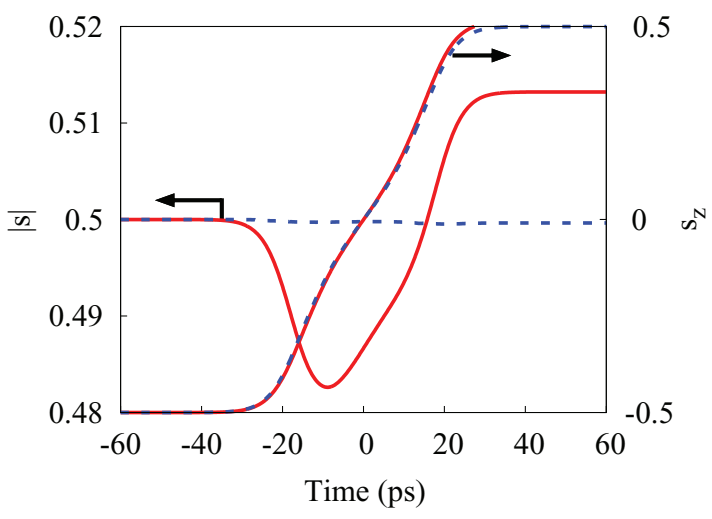

FIG. 9. (Color online) Comparison of the dynamics obtained from the Lindblad [Eq. (7), dashed curves] and non-Lindblad [Eq. (4), solid curves] forms of time-dependent Markovian approximations, for the Gaussian ARP pulse, Eq. (12), with $\tau_{0}=2 \mathrm{ps}, T=1 \mathrm{~K}$, $a=30 \mathrm{ps}^{2}$, and $\Theta_{0}=5 \pi$. For each approach, both the magnitude of the pseudospin (Bloch) vector $|s|$ (left axis) and inversion $s_{z}$ (right axis) are shown. At this low temperature, as discussed above, the Markov approximation holds reasonably well, yet the non-Lindblad form leads to unphysical results $|s|,\left|s_{z}\right|>0.5$. 

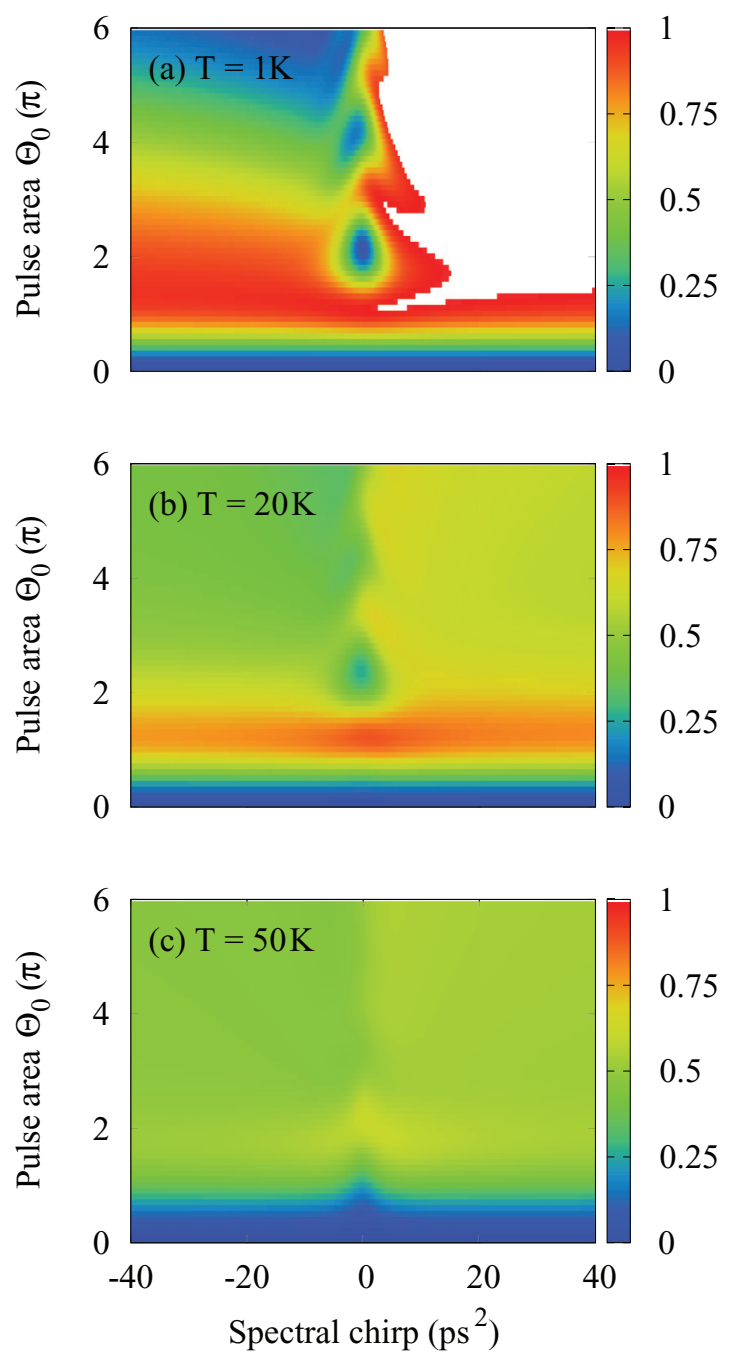

FIG. 10. (Color online) Final exciton occupation probability following an ARP pulse of the form given in Eq. (12), calculated using the non-Lindblad form [see Eq. (4)], for comparison with the results obtained from the Lindblad form [see Eq. (7)] shown in Fig. 4. The parameters and scale are as in Fig. 4. The results are qualitatively similar for many parameters. However, there is a significant region in the top panel (white) where the density operator obtained from Eq. (4) does not remain positive, and the results become unphysical.

[which shows the results of the Lindblad Eq. (7)]. Despite this similarity for much of the parameter range, Eq. (7) is required to produce valid results across the problems considered in the current paper.

There have been suggestions that time-dependent densitymatrix equations, which are not of the Lindblad form, such as those derived from the time-convolutionless (TCL) approach, ${ }^{12}$ may in some cases ensure positivity ${ }^{44}$ However, Eq. (4) is essentially the result of the time-convolutionless approach at second order (TCL2). The only difference is that for TCL2, the lower limit on integrals over $t^{\prime}$ such as Eq. (6) is $t^{\prime}=0$, rather than $t^{\prime}=-\infty$, corresponding to starting the system at $t=0$ in a factorized state. Even for time independent $\Delta$ and $\Omega$, this means the coefficients in the density matrix evolution become time dependent, but eventually decay toward a steady-state value. As such, for the ARP pulse, as long as the pulse duration is long compared to the bath correlation time, these additional time dependencies die out, and TCL2 becomes equivalent to the positivity-violating form in Eq. (4).

\section{CONCLUSIONS}

In conclusion, we have shown how the Bloch-RedfieldWangsness theory may be used to derive a time-dependent Lindblad form describing the dephasing of quantum-dot excitons by acoustic phonons in the presence of a driving laser field. We have outlined the application of this theory to recent ARP experiments ${ }^{4,5}$ on single quantum dots and predict, in agreement with numerical work, ${ }^{9}$ that phonons have a pronounced effect on ARP even at cryogenic temperatures. Their effect can, however, be almost eliminated by an appropriate choice of pulse shape. Furthermore, this pulse-shape dependence could allow forms of phonon spectroscopy based on ARP pulses or on off-resonant continuous-wave excitation. More generally, our approach captures the physics of dynamical, excitation-controlled dephasing in which the driving field changes the eigenspectrum of the dot, and hence the decoherence and scattering rates. The resulting Lindblad form is straightforward to simulate, gives qualitatively reasonable results over wide parameter regimes, and is expected to be quantitatively accurate at low temperatures for slow pulses. It can be applied to a wide variety of pulse sequences, and the approach is generalized to address a wide range of problems relating to the decoherence of solid-state qubits, such as the limitations on the creation of entangled states in coupled quantum dots, ${ }^{45-49}$ the persistence of entanglement in interacting solid-state systems, and the emission spectra of solid-state qubits in the strong-coupling regime..$^{50,51}$

\section{ACKNOWLEDGMENTS}

PRE acknowledges support from Science Foundation Ireland (09/SIRG/I1592). AOS acknowledges funding from the Carnegie Trust for the Universities of Scotland and the St. Andrews Undergraduate Research Internship Program. JK acknowledges useful discussions with B. W. Lovett, F. B. F. Nissen, P. Öhberg, and support from EPSRC (EP/G004714/2 and $\mathrm{EP} / \mathrm{I} 031014 / 1)$.

\section{APPENDIX: DERIVATION OF SECULARIZED DENSITY MATRIX EQUATION}

This appendix provides further details of the derivation of the time-dependent Markovian Lindblad form in Eq. (7). Following the usual approach, ${ }^{12}$ working in the interaction picture, the effects of the system-bath coupling on the system density matrix can be included to second order by writing

$$
\dot{\tilde{\rho}}=-\int_{-\infty}^{t} d t^{\prime} \operatorname{Tr}_{B}\left[\tilde{H}_{c}(t),\left[\tilde{H}_{c}\left(t^{\prime}\right), \tilde{\rho}\left(t^{\prime}\right) \otimes \tilde{\rho}_{B}(0)\right]\right],
$$

where tilde indicates the interaction picture, $\tilde{\rho}_{B}$ is the phonon bath density matrix, and $H_{c}$ is given in Eq. (2).

In the case that $\Delta$ and $\Omega$ vary slowly with time, we may effectively transform the coupling Hamiltonian to the interaction picture by using the instantaneous eigenstates 
(dressed states) giving

$$
\begin{aligned}
H_{\mathrm{c}}(t) & =P(t) \Phi(t) \\
& =e^{i t H_{\mathrm{dot}}} s_{z} e^{-i t H_{\mathrm{dot}}} \sum_{q}\left(g_{q} b_{q} e^{-i v_{q} t}+g_{q}^{*} b_{q}^{\dagger} e^{i v q}\right),
\end{aligned}
$$

where $v_{q}$ is the phonon frequency. We transform to the instantaneous dressed states, defining spin operators $\mathbf{r}=R \mathbf{s}$, with $R$ a rotation by angle $\tan ^{-1} \Omega / \Delta$ around the $y$ axis. Then the coupling operators become

$$
P(t)=\frac{\Delta}{\Lambda} r_{z}+\frac{\Omega}{2 \Lambda}\left(r_{+} e^{i \Lambda t}+r_{-} e^{-i \Lambda t}\right),
$$

where $\Lambda=\sqrt{\Omega^{2}+\Delta^{2}}$ is the splitting of the instantaneous eigenstates and $r_{ \pm}$cause transitions between these states.

Note that for a time-dependent Hamiltonian, the true interaction-picture form is obtained with a unitary transformation involving a time-ordered exponential, $U=$ $T e^{-i \int^{t} H_{\mathrm{dot}}\left(t^{\prime}\right) d t^{\prime}}$, and this operator is not generally approximated by the form in Eq. (A2). However, in the following, we use the form in Eq. (A2) only to calculate the effects of phonons on short time scales, $t_{c} \sim 1 / \omega_{c}$, with the final equation for the density matrix obtained by undoing this formal transformation. Thus we expect that the Hamiltonian part of the dynamics is not approximated in the result, while the dissipative part is correct provided $t_{c} d \Delta / d t, t_{c} d \Omega / d t \ll \Lambda$. These conditions are well satisfied for the protocols considered in this paper.

With these forms of $P(t)$ and $\Phi(t)$, we may then follow the normal steps of tracing over the phonon bath to give the system density matrix equation:

$$
\begin{aligned}
\dot{\tilde{\rho}}= & -\int d v J(v) \int^{t} d t^{\prime}\left\{\left[P(t) P\left(t^{\prime}\right) \tilde{\rho}\left(t^{\prime}\right)-P\left(t^{\prime}\right) \tilde{\rho}\left(t^{\prime}\right) P(t)\right]\right. \\
& \times\left[\left(n_{v}+1\right) e^{-i v\left(t-t^{\prime}\right)}+n_{\nu} e^{i v\left(t-t^{\prime}\right)}\right] \\
& -\left[P(t) \tilde{\rho}\left(t^{\prime}\right) P\left(t^{\prime}\right)-\tilde{\rho}\left(t^{\prime}\right) P\left(t^{\prime}\right) P(t)\right] \\
& \left.\times\left[\left(n_{v}+1\right) e^{i v\left(t-t^{\prime}\right)}+n_{v} e^{-i v\left(t-t^{\prime}\right)}\right]\right\}
\end{aligned}
$$

where $n_{v}$ is the thermal occupation of the phonons at frequency $v$. After performing the integrations over frequency, the remaining integral contains factors that are sharply peaked functions of $t-t^{\prime}$, decaying over a time scale $1 / \omega_{c}$. $\tilde{\rho}\left(t^{\prime}\right)$, $\Delta\left(t^{\prime}\right)$, and $\Omega\left(t^{\prime}\right)$ vary little over this time scale, and so may be approximated by their values at $t$. However, $P\left(t^{\prime}\right)$ may vary over this time scale due to the time-dependence arising from the transformation to the interaction picture. If we approximate $\tilde{\rho}\left(t^{\prime}\right) \simeq \tilde{\rho}(t)$ and perform no other steps, this leads to Eq. (4), which as noted before, is not of Lindblad form.

Following Dumcke and Spohn, ${ }^{37}$ the corresponding Lindblad form arises by "secularizing" Eq. (A4). This corresponds to averaging the above equation over a time short compared to decay rates, but long compared to the time scales of the system Hamiltonian - the fact that such a time scale exists is implicit in the use of a perturbative (Born) approximation. We start from Eq. (4), with $P(t)$ as defined in Eq. (5), and writing $Q(t)$ in the generic form:

$$
Q(t)=\Gamma_{z} r_{z}+\Gamma_{+} r_{+} e^{i \Lambda t}+\Gamma_{-} r_{-} e^{-i \Lambda t} .
$$

This follows directly from performing the integrals in Eq. (6), and so $\Gamma_{z}, \Gamma_{ \pm}$are various frequency integrals over $J(v)$. Multiplying $P(t)$ and $Q(t)$ and integrating over a time long compared to $1 / \Lambda$, only those terms with equal and opposite $t$ dependence will survive, i.e., those terms involving $r_{z} r_{z}, r_{+} r_{-}$ or $r_{-} r_{+}$. The secularized Eq. (4) thus becomes

$$
\begin{aligned}
\dot{\tilde{\rho}}= & \frac{\Delta}{\Lambda}\left(\Gamma_{z}+\Gamma_{z}^{*}\right)\left(r_{z} \tilde{\rho} r_{z}-\tilde{\rho}\right) \\
& -\frac{\Omega}{2 \Lambda}\left[\Gamma_{+}\left(r_{-} r_{+} \tilde{\rho}-r_{+} \tilde{\rho} r_{-}\right)+\Gamma_{+}^{*}\left(\tilde{\rho} r_{-} r_{+}-r_{+} \tilde{\rho} r_{-}\right)\right] \\
& -\frac{\Omega}{2 \Lambda}\left[\Gamma_{-}\left(r_{+} r_{-} \tilde{\rho}-r_{-} \tilde{\rho} r_{+}\right)+\Gamma_{-}^{*}\left(\tilde{\rho} r_{+} r_{-}-r_{-} \tilde{\rho} r_{+}\right)\right] .
\end{aligned}
$$

The vanishing of the phonon density of states as $\omega \rightarrow 0$ ensures that $\Gamma_{z}+\Gamma_{z}^{*}=0$, and the remaining terms take the form of the Lindblad decay and phonon Lamb shift terms as given in Eq. (7).
${ }^{1}$ A. N. Vamivakas and M. Atatüre, Contemp. Phys. 51, 17 (2010).

${ }^{2}$ T. H. Stievater, X. Li, D. G. Steel, D. Gammon, D. S. Katzer, D. Park, C. Piermarocchi, and L. J. Sham, Phys. Rev. Lett. 87, 133603 (2001).

${ }^{3}$ A. J. Ramsay, Semicond. Sci. Technol. 25, 103001 (2010),

${ }^{4}$ Y. Wu, I. M. Piper, M. Ediger, P. Brereton, E. R. Schmidgall, P. R. Eastham, M. Hugues, M. Hopkinson, and R. T. Phillips, Phys. Rev. Lett. 106, 067401 (2011).

${ }^{5}$ C.-M. Simon, T. Belhadj, B. Chatel, T. Amand, P. Renucci, A. Lemaitre, O. Krebs, P. A. Dalgarno, R. J. Warburton, X. Marie, and B. Urbaszek, Phys. Rev. Lett. 106, 166801 (2011).

${ }^{6}$ E. R. Schmidgall, P. R. Eastham, and R. T. Phillips, Phys. Rev. B 81, 195306 (2010).

${ }^{7}$ P. R. Eastham and R. T. Phillips, Phys. Rev. B 79, 165303 (2009).

${ }^{8}$ A. J. Ramsay, A. V. Gopal, E. M. Gauger, A. Nazir, B. W. Lovett, A. M. Fox, and M. S. Skolnick, Phys. Rev. Lett. 104, 017402 (2010). ${ }^{9}$ S. Lüker, K. Gawarecki, D. E. Reiter, A. Grodecka-Grad, V. M. Axt, P. Machnikowski, and T. Kuhn, Phys. Rev. B 85, 121302 (2012).
${ }^{10}$ D. E. Reiter, S. Lüker, K. Gawarecki, A. Grodecka-Grad, P. Machnikowski, V. M. Axt, and T. Kuhn, Acta Phys. Pol. A 122, 1065 (2012).

${ }^{11}$ K. Schuh, F. Jahnke, and M. Lorke, Appl. Phys. Lett. 99, 011105 (2011).

${ }^{12}$ H.-P. Breuer and F. Petruccione, The Theory of Open Quantum Systems (Oxford University Press, Oxford, 2007).

${ }^{13}$ I. Wilson-Rae and A. Imamoğlu, Phys. Rev. B 65, 235311 (2002).

${ }^{14}$ A. Vagov, M. D. Croitoru, V. M. Axt, T. Kuhn, and F. M. Peeters, Phys. Rev. Lett. 98, 227403 (2007).

${ }^{15}$ A. J. Ramsay, T. M. Godden, S. J. Boyle, E. M. Gauger, A. Nazir, B. W. Lovett, A. M. Fox, and M. S. Skolnick, Phys. Rev. Lett. 105, 177402 (2010)

${ }^{16}$ D. P. S. McCutcheon, N. S. Dattani, E. M. Gauger, B. W. Lovett, and A. Nazir, Phys. Rev. B 84, 081305 (2011).

${ }^{17}$ J. Förstner, C. Weber, J. Danckwerts, and A. Knorr, Phys. Rev. Lett. 91, 127401 (2003). 
${ }^{18}$ A. J. Ramsay, T. M. Godden, S. J. Boyle, E. M. Gauger, A. Nazir, B. W. Lovett, A. V. Gopal, A. M. Fox, and M. S. Skolnick, J. Appl. Phys. 109, 102415 (2011).

${ }^{19}$ D. P. S. McCutcheon and A. Nazir, New J. Phys. 12, 113042 (2010).

${ }^{20}$ A. Nazir, Phys. Rev. B 78, 153309 (2008).

${ }^{21}$ J. D. Cresser, J. Mod. Opt. 39, 2187 (1992).

${ }^{22}$ C. Roy and S. Hughes, Phys. Rev. Lett. 106, 247403 (2011).

${ }^{23}$ C. Roy and S. Hughes, Phys. Rev. B 85, 115309 (2012).

${ }^{24}$ C. Roy, H. Kim, E. Waks, and S. Hughes, Phot. Nano: Fund. Appl. 10, 359 (2012).

${ }^{25}$ S. Hughes and H. J. Carmichael, arXiv:1210.0488.

${ }^{26}$ R. Silbey and R. A. Harris, J. Chem. Phys. 80, 2615 (1984).

${ }^{27}$ R. K. Wangsness and F. Bloch, Phys. Rev. 89, 728 (1953).

${ }^{28}$ A. G. Redfield, Phys. Rev. 98, 1787 (1955).

${ }^{29}$ M. Glässl, A. Vagov, S. Lüker, D. E. Reiter, M. D. Croitoru, P. Machnikowski, V. M. Axt, and T. Kuhn, Phys. Rev. B 84, 195311 (2011).

${ }^{30}$ A. Vagov, M. D. Croitoru, V. M. Axt, P. Machnikowski, and T. Kuhn, Phys. Status Solidi B 248, 839 (2011).

${ }^{31}$ C. Duke and G. Mahan, Phys. Rev. 139, A1965 (1965).

${ }^{32}$ G. D. Mahan, Many-Particle Physics (Plenum Press, New York, 2000).

${ }^{33}$ G. W. Ford and R. F. O'Connell, Phys. Rev. Lett. 77, 798 (1996).

${ }^{34}$ G. W. Ford and R. F. O'Connell, Phys. Rev. Lett. 82, 3376 (1999).

${ }^{35}$ M. Lax, Opt. Commun. 179, 463 (2000).

${ }^{36}$ G. Ford, Opt. Commun. 179, 451 (2000).
${ }^{37}$ R. Dumcke and H. Spohn, Z. Phys. B 34, 419 (1979).

${ }^{38}$ H. Spohn, Rev. Mod. Phys. 52, 569 (1980).

${ }^{39}$ T. M. Stace, A. C. Doherty, and S. D. Barrett, Phys. Rev. Lett. 95, 106801 (2005).

${ }^{40}$ E. M. Gauger, S. C. Benjamin, A. Nazir, and B. W. Lovett, Phys. Rev. B 77, 115322 (2008).

${ }^{41}$ V. S. Malinovsky and J. L. Krause, Eur. Phys. J. D 14, 147 (2001).

${ }^{42}$ S. Guérin, S. Thomas, and H. R. Jauslin, Phys. Rev. A 65, 023409 (2002).

${ }^{43}$ K. Wen, T. Byrnes, and Y. Yamamoto, arXiv:1210.0173.

${ }^{44}$ R. S. Whitney, J. Phys. A: Math. Theor. 41, 175304 (2008).

${ }^{45}$ C. Creatore, R. T. Brierley, R. T. Phillips, P. B. Littlewood, and P. R. Eastham, Phys. Rev. B 86, 155442 (2012).

${ }^{46}$ R. G. Unanyan, N. V. Vitanov, and K. Bergmann, Phys. Rev. Lett. 87, 137902 (2001).

${ }^{47}$ Z. Kis and E. Paspalakis, J. Appl. Phys. 96, 3435 (2004).

${ }^{48}$ U. Hohenester, J. Fabian, and F. Troiani, Opt. Commun. 264, 426 (2006).

${ }^{49}$ S. K. Saikin, C. Emary, D. G. Steel, and L. J. Sham, Phys. Rev. B 78, 235314 (2008).

${ }^{50}$ Y. Makhlin, G. Schön, and A. Shnirman, Rev. Mod. Phys. 73, 357 (2001).

${ }^{51}$ P. Kaer, T. R. Nielsen, P. Lodahl, A.-P. Jauho, and J. Mørk, Phys. Rev. Lett. 104, 157401 (2010). 